\title{
The three-dimensional Anderson model of localization with binary random potential
}

\author{
I V Plyushchay $\dagger^{\ddagger}$, R A Römer $\ddagger^{\S}$ and M Schreiber $\boldsymbol{\Phi}^{* *}$ \\ $\dagger$ †epartment of Physics, Kiev Taras Shevchenko National University, Volodymyrska st. 64, Kiev, 01033 Ukraine \\ $\ddagger$ Department of Physics, University of Warwick, Coventry, CV4 7AL, United Kingdom and \\ \Institut für Physik, Technische Universität, D-09107 Chemnitz, Germany
}

(Dated: Revision : 1.37, compiled October 23, 2018)

\begin{abstract}
We study the three-dimensional two-band Anderson model of localization and compare our results to experimental results for amorphous metallic alloys (AMA). Using the transfer-matrix method, we identify and characterize the metal-insulator transitions as functions of Fermi level position, band broadening due to disorder and concentration of alloy composition. The appropriate phase diagrams of regions of extended and localized electronic states are studied and qualitative agreement with AMA such as $\mathrm{Ti}-\mathrm{Ni}$ and $\mathrm{Ti}-\mathrm{Cu}$ metallic glasses is found. We estimate the critical exponents $\nu_{W}, \nu_{E}$ and $\nu_{x}$ when either disorder $W$, energy $E$ or concentration $x$ is varied, respectively. All our results are compatible with the universal value $\nu \approx 1.6$ obtained in the single-band Anderson model.
\end{abstract}

PACS numbers: 71.23.An, 71.30.+h, 71.55.Jv

\section{INTRODUCTION}

Amorphous metallic alloys (AMA) offer the possibility of continuously changing their composition while at the same time avoiding structural phase transformations. Thus they allow for systematic studies of their physical properties within a single phase as temperature and other external control parameters, e.g., pressure, are varied. Many such investigations (see, e.g., [1]) have been devoted to the investigation of the electrophysical properties (in particular, electrical resistivity) of AMA; these studies have revealed considerable differences in the behavior of the electrical resistivity $\varrho$ of AMA in comparison with that of their crystalline counterparts.

The transport properties of transition-metal-based AMA are of special interest because of unique physical anomalies, unexplained by conventional transport theory, that exist in these materials. Among them are the negative temperature coefficient of resistivity, the Mooij correlation of resistivity and thermoelectric power [2] - the higher the resistivity the lower is the temperature coefficient of the resistivity - , the resistivity saturation, the sign reversal of the Hall coefficient, a negative magnetoresistance, and the breakdown of Mattiessen's rule. For a review, we refer the reader to ref. [3]. The first attempt for a theoretical understanding of the electronic transport in noncrystalline materials was the Ziman theory and its extensions [1]. However, the applicability of the Ziman diffraction model for strongly disordered and thus high-resistivity amorphous alloys is questionable [4, [5]. In addition, the Ziman theory cannot explain, e.g., that the changes of the resistivity $\left(\varrho_{\text {liquid }}-\varrho_{\text {solid }}\right) / \varrho_{\text {solid }}$ at the melting point are only 0.01 and 0.09 for $\mathrm{Fe}$ and $\mathrm{Co}$, respectively [1]. Thus it appears that the resistivity of

\footnotetext{
‡innapl@univ.kiev.ua

$\S_{\text {r.roemer@warwick.ac.uk }}$

**schreiber@physik.tu-chemnitz.de
}

these two elements depends only weakly on their atomic structure factor $S(k)$.

At low temperature $T$, an even more significant difference between the behavior of crystals on the one hand and disordered solids on the other hand is seen: sufficiently strong disorder can give rise to a transition of the transport properties from conducting behavior with resistance $R>0$ to insulating behavior with $R=\infty$ as $T \rightarrow 0$ as was pointed out by Anderson in 1958 [6] , This phenomena is called the disorder-driven metal-insulator transition (MIT) [3, 7, 8] and it is characteristic to noncrystalline solids. The mechanism underlying this MIT was attributed by Anderson not to a finite gap in the energy spectrum which is responsible for an MIT in band gap or Mott insulators 9]. Rather, he argued that the disorder will lead to interference of the electronic wave function $\psi(\mathbf{r})$ with itself such that it is no longer extended over the whole solid but is instead confined to a small part of the solid. This localization effect excludes the possibility of diffusion at $T=0$ so that the system is an insulator. A highly successful theoretical approach to this disorderinduced MIT was put forward in 1979 by Abrahams et al. [10]. This "scaling hypothesis of localization" details the existence of an MIT for non-interacting electrons in three-dimensional disordered systems at zero magnetic field and in the absence of spin-orbit coupling.

These ideas have also been applied to the analysis of AMA transport properties 11]. They provide a background for an explanation of the Mooij correlation 12 and were successful in the description of the transport properties of amorphous semiconductors 13. Many papers have since discussed the influence of localization (or quantum interference effects) on the transport properties of AMA 14, 15, 16, 17]. It was shown 18 that Anderson localization is responsible for regions of high resistivity. The importance of quantum corrections in analyzing the conductivity of so-called highly resistive $(\varrho>150 \mu \Omega \mathrm{cm})$ metallic glasses was demonstrated [19]. There are two sources of these corrections: the disorder-induced "localization effect" and the electronic "interaction effect". 
The former caused the observed higher temperature resistivity. Furthermore, it has been argued [20, 21] that for amorphous metals localization effects are valid even at room temperature.

In a recent study 22] the influence of disorder on the transport properties of $\mathrm{Ti}-\mathrm{Ni}$ and $\mathrm{Ti}-\mathrm{Cu}$ metallic glasses was analyzed within the Anderson model of localization. The results elucidated qualitatively the correlation between the observed details of the electronic structure and the behavior of resistivity vs. temperature for this binary AMA. It was shown that the temperature coefficient of resistivity depends on the position of the Fermi level $E_{\mathrm{F}}$ and on the (assumed) position of the mobility edge $E_{\mathrm{c}}$. The latter separates extended (conducting) states from localized (insulating) states 8, 23]. Unfortunately, the value of $E_{\mathrm{c}}$ had to be inferred from the hypothesis that the two subbands of the valence band of the binary alloy have tails with localized electronic states like the tails of the usual one-band Anderson model [ $[$ ]. Evidently, a calculation of the position of the mobility edge $E_{\mathrm{c}}$ should be carried out for the case of two subbands. This is what we intend to do in the present manuscript. In particular, we will investigate whether localized electronic states exist in the central region between the two subbands as assumed in 22]. Furthermore, we show how the concentration parameter, absent in the usual single-band Anderson model, influences the position of the mobility edges.

\section{NUMERICAL APPROACH}

\section{A. The binary Anderson model of localization}

According to the photo-emission data and theoretical estimates [22, 24], the valence band of a binary, transition-metal-based amorphous alloy can be assumed to be a superposition of two valence bands $A$ and $B$. In order to model this within the Anderson model, we use the standard Anderson Hamiltonian [ $[\underline{6}$

$$
\mathbf{H}=\sum_{i} \varepsilon_{i}|i\rangle\left\langle i\left|+\sum_{i \neq j} t_{i j}\right| i\right\rangle\langle j|
$$

with orthonormal states $|i\rangle$ corresponding to electrons located at sites $i=(x, y, z)$ of a regular cubic lattice with periodic boundary conditions. The hopping integrals $t_{i j}$ are non-zero only for nearest neighbors and we set the energy scale by choosing $t_{i j}=t=1$. The twosubband structure observed in the experiments is incorporated into the potential energies $\varepsilon_{i}$. The randomness is modeled by

1. independent random variations $\varepsilon_{i(A)} \in\left[\varepsilon_{A}-\right.$ $\left.W_{A} / 2, \varepsilon_{A}+W_{A} / 2\right]$ and $\varepsilon_{i(B)} \in\left[\varepsilon_{B}-W_{B} / 2, \varepsilon_{B}+\right.$ $\left.W_{B} / 2\right]$,

2. random spatial distribution of potential energies $\varepsilon_{A}$ and $\varepsilon_{B}$ within the cubic lattice.
The mean values $\varepsilon_{A}$ and $\varepsilon_{B}$ are chosen according to the central energies of the two subbands. The parameters $W_{A}$ and $W_{B}$ specify the disorder strength in each energy band and the random variation of $A$ and $B$ sites models the compositional disorder of the binary alloy. Note that with this choice of parameters, the band edges of the subband $A$ in the limit of large system size are given by $\varepsilon_{A}-6-W_{A} / 2, \varepsilon_{A}+6+W_{A} / 2$ and similarly for the other subband with $A \rightarrow B$.

In summary, the model is described by the following parameters

1. $\varepsilon_{A B}=\varepsilon_{B}-\varepsilon_{A}$, the distance between the centers of the two bands (we choose $\varepsilon_{B}>\varepsilon_{A}$ so that the $A$ band is the lower one),

2. $x_{A}$, the concentration of $A$ sites (of course, then $x_{B}=1-x_{A}$ is the concentration of the $B$ sites),

3. $W_{A}$ and $W_{B}$, the widths of the box distributions of the potential energy.

\section{B. Transfer-matrix method}

Since we are interested in the position of the mobility edges and thus the localization lengths, we use the transfer-matrix method (TMM) $[8,25,26]$ for the investigation of model (11). The localization length $\lambda$ describes the exponential decay of the wave function and we compute it using TMM for quasi-1D bars of cross section $M \times M$ and length $L \gg M$. As is customary in the TMM approach, the stationary Schrödinger equation $\mathbf{H} \Psi=E \Psi$ is rewritten in the recursive form

$$
\left(\begin{array}{c}
\Psi_{i+1} \\
\Psi_{i}
\end{array}\right)=\left(\begin{array}{cc}
E \mathbf{1}-\mathbf{H}_{i} & -\mathbf{1} \\
\mathbf{1} & \mathbf{0}
\end{array}\right)\left(\begin{array}{c}
\Psi_{i} \\
\Psi_{i-1}
\end{array}\right)=\mathbf{T}_{i}\left(\begin{array}{c}
\Psi_{i} \\
\Psi_{i-1}
\end{array}\right) .
$$

$\Psi_{i}, \mathbf{H}_{i}$, and $\mathbf{T}_{i}$ are wave function, Hamiltonian matrix, and transfer matrix of the $i$ th slice of the bar, respectively. Unit and zero matrices are denoted by $\mathbf{1}$ and $\mathbf{0}$. Given an initial condition $\left(\begin{array}{l}\Psi_{1} \\ \Psi_{0}\end{array}\right)$ equation (2) allows a recursive construction of the wave function in the bar geometry by adding more and more slices. $\lambda(M, w)$ is then obtained from the smallest Lyapunov exponent of the product $\mathbf{T}_{L} \mathbf{T}_{L-1} \cdots \mathbf{T}_{2} \mathbf{T}_{1}$ of transfer matrices [27], where the length $L$ of the bar is increased until the desired accuracy of $\lambda$ is achieved. With increasing cross section of the bar the reduced localization length $\Lambda_{M}(w)=\lambda(M, w) / M$ decreases for localized states and increases for extended states. Thus it is possible to determine the critical parameter $w_{\mathrm{c}}$ at which $\Lambda_{M}$ is constant as a function of the varied parameter $w-$ e.g., $E, \varepsilon_{A B}, x_{A}, W_{A}$, or $W_{B}-$ from plots of $\Lambda_{M}$ versus $M$.

\section{Finite-size scaling}

The MIT in the Anderson model of localization is expected to be a second-order phase transition [7, 10]. It is 
characterized by a divergent correlation length $\xi_{\infty}(w)=$ $C\left|w-w_{\mathrm{c}}\right|^{-\nu}$, where $\nu$ is the critical exponent, $C$ is a constant [8], $w$ is any of the external control parameters given above and $w_{\mathrm{c}}$ is its critical value at which the MIT occurs. To construct the correlation length of the infinite system $\xi_{\infty}$ from finite-size data $\Lambda_{M}[8,25,26,28]$, the one-parameter scaling hypothesis [29] is employed,

$$
\Lambda_{M}=f\left(M / \xi_{\infty}\right) .
$$

All values of $\Lambda_{M}(w)$ are expected to collapse onto a single scaling curve $f$, when the system size is scaled by $\xi_{\infty}(w)$. In a system with MIT such a scaling curve consists of two branches corresponding to the localized and the extended phase. One might determine $\nu$ from fitting $\xi_{\infty}$ by a finite-size scaling (FSS) procedure [27]. But a higher accuracy can be achieved by fitting directly the raw data [27. We use fit functions [30] which include two kinds of corrections to scaling: (i) nonlinearities of the $w$ dependence of the scaling variable and (ii) an irrelevant scaling variable with exponent $-y$. Specifically, we fit

$$
\begin{gathered}
\Lambda_{M}=\tilde{f}_{0}\left(\chi_{\mathrm{r}} M^{1 / \nu}\right)+M^{-y} \tilde{f}_{1}\left(\chi_{\mathrm{r}} M^{1 / \nu}\right) \\
\tilde{f}_{n}=\sum_{i=0}^{n_{\mathrm{r}}} a_{n i} \chi_{\mathrm{r}}^{i} M^{i / \nu} \quad, \quad \chi_{\mathrm{r}}(\omega)=\omega+\sum_{n=2}^{m_{\mathrm{r}}} b_{n} \omega^{n}
\end{gathered}
$$

with $\omega=\left|w_{\mathrm{c}}-w\right| / w_{\mathrm{c}}$ and expansion coefficients $a_{n i}$ and $b_{n}$. Choosing the orders $n_{\mathrm{r}}$ and $m_{\mathrm{r}}$ of the expansions larger than one, terms with higher order than linear in the $w$ dependence appear. This allows to fit a wider $w$ range around $w_{\mathrm{c}}$ than with the previously used linear fitting [31]. The linear region is usually very small. The second term in equation (4) describes the systematic shift of the crossing point of the $\Lambda_{M}(w)$ curves 30,31 .

In the present case of the two-band Anderson model, we can in principle have an MIT as a function of

1. energy $E$ for fixed $W_{A}, W_{B}, \varepsilon_{A B}$ and $x_{A}$,

2. disorder strengths $W_{A}, W_{B}$ for fixed $E, \varepsilon_{A B}$ and $x_{A}$,

3. concentration $x_{A}$ for fixed $E, \varepsilon_{A B}$ and $W_{A}, W_{B}$.

Due to universality, we expect the corresponding critical exponents $\nu_{E}, \nu_{W_{A}}, \nu_{W_{B}}$ and $\nu_{x}$ to be the same. Additionally for each control parameter we test whether the fitted values of $w_{\mathrm{c}}$ and $\nu$ are compatible when using different expansions of the fit function, i.e., different orders $n_{\mathrm{r}}$ and $m_{\mathrm{r}}$ 32].

\section{RESULTS AND DISCUSSION}

For the determination of the mobility edge [23], we have determined the localization lengths from TMM with an error equal to or less than $1 \%$ for cross-sections up to $M=12$. For the computation of the critical exponents,

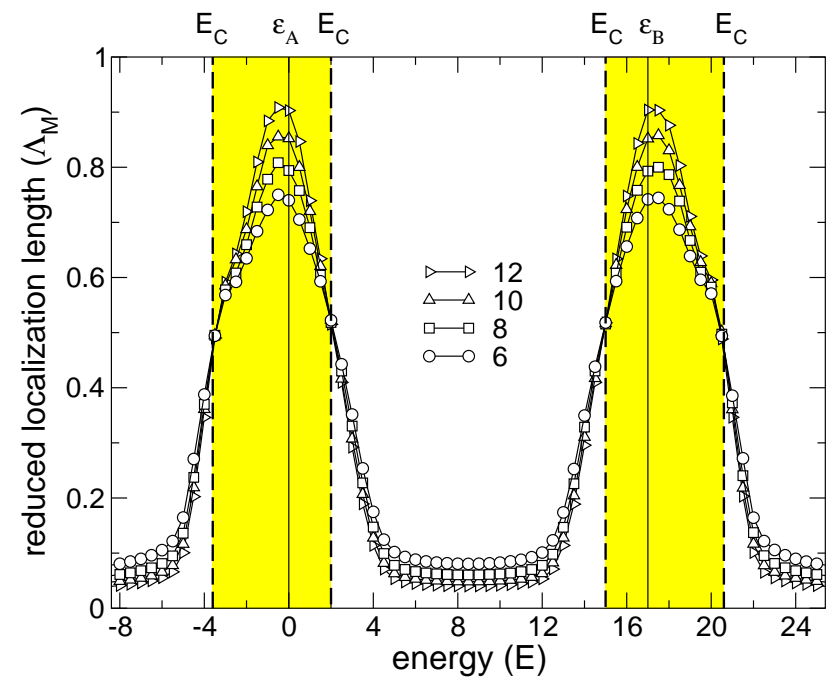

FIG. 1: Dependence of the reduced localization length $\Lambda_{M}$ on energy $E$ for different system sizes $M=6,8,10$ and 12 at $\varepsilon_{A B}=17, x_{A}=0.5$ and $W_{A}=W_{B}=5$. The two shaded regions indicate extended states, the dashed lines the position of the 4 mobility edges, and the vertical solid lines the band centres of the subbands. No error bars are shown because the accuracy of the $\Lambda_{M}$ values is better than the size of the symbols.

we have used the available $1 \%$ data and generated additional data with $0.05 \%$ error up to at most $0.1 \%$ at selected points in the phase diagrams for high-precision estimates.

In Fig. 10 we show a typical dependence of the reduced localization length $\Lambda_{M}$ on energy for different system sizes $M$. The two peaks correspond roughly to the positions of the two subbands at $\varepsilon_{A}$ and $\varepsilon_{B}$, respectively. This is in accordance with the theoretical and experimental results 22, 24] mentioned in the introduction as motivation of our studies. Note that for the chosen parameters, the density-of-states limit given in section IIA for the highest energy state in the lower subband coincides with the smallest possible energy state in the upper subband at $\varepsilon_{A}+6+W_{A} / 2=\varepsilon_{B}-6-W_{B} / 2$. At the positions indicated by the dashed lines in Fig. 10 we see a reversal in the systematic size dependence of the reduced localization lengths. Therefore these indicate the mobility edges and we observe localized electronic states at the band edges and in the central region between the $A$ and $B$ subbands. We remark that the $\Lambda_{M}(E)$ curves demonstrate a peculiar feature: the positions of the maximum values of $\Lambda_{M}$ do not coincide with the central energies $\varepsilon_{A}$ and $\varepsilon_{B}$ of the two subbands. Thus although the density of states between the two bands is appreciable, we observe the surprising fact that the region of extended states in each band has shrunk.

In Fig. 2] we show high precision data at the upper mobility edge of the $A$ subband at $E_{\mathrm{c}} \approx 2$. Again the accuracy of the data is so high that error bars would 


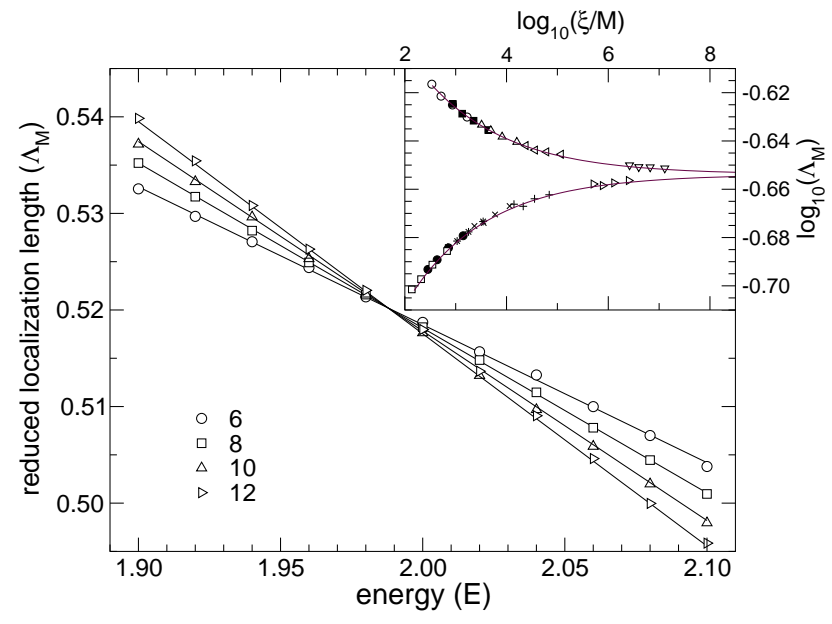

FIG. 2: FSS plot of the reduced localization length $\Lambda_{M}$ near the mobility edge $E_{\mathrm{c}} \approx 2$ of Fig. 1] The solid lines are fits of the data according to equations (4) and (5) with $n_{\mathrm{r}}=1$ and $m_{\mathrm{r}}=1$. The inset shows the scaling function corresponding to the fit and the $\Lambda_{M}$ data with symbols $\circ, \mathbf{\square}, \triangle, \triangleleft, \nabla, \triangleright,+, *, \bullet, \square$ denoting energies $1.9,1.92,1.94, \ldots, 2.08,2.10$, respectively.

be smaller than the symbols. Data and FSS analysis of similar quality will be used when estimating critical exponents in the following.

\section{A. Energy-disorder phase diagram}

Let us now investigate how the positions of the mobility edges change when $W_{A}$ and $W_{B}$ are changed. We set $\varepsilon_{A B}=17$ and $x_{A}=x_{B}=0.5$. For convenience we choose $W_{A} \equiv W_{B}$. This leads to a symmetry for the energy dependences of $\Lambda_{M}(E)$ between lower and upper subband as shown in Fig. 1 Consequently, the phase diagram is symmetric with respect to $\varepsilon_{A}+\varepsilon_{A B} / 2$. We find extended electronic states in the vicinity of $\varepsilon_{A}$ and for $W_{A} \leq W_{\mathrm{c}} \approx 6.5$ (instead of 16.5 as for the usual Anderson model) as presented in Fig. 3. For larger $W_{A}$ or larger $\left|E-\varepsilon_{A}\right|$, the states are localized.

Next, we keep $W_{A}=4$ fixed and vary $W_{B}$ from 0 to 8. We find for $\varepsilon_{A B}=17$ that the position of mobility edges in the subband $A$ is only slightly modified. The same behavior is observed for $\varepsilon_{A B}=8$, when the overlap of the two subbands is large.

\section{B. Energy- $\varepsilon_{A B}$ phase diagram}

Keeping $x_{A}=0.5$ and fixed disorder $W_{A}=W_{B}=4$, we now vary the subband spacing $\varepsilon_{A B}$. From an experimental point of view, this is the difference between the atomic constituents of the alloy or, more precisely, between their ionization energies. For a variety of $\varepsilon_{A B}$ values we have obtained $\Lambda_{M}$ curves which are qualitatively very similar to Fig. [1 However, for $\varepsilon_{A B} \leq 6$, no localized electronic states in the central region can be

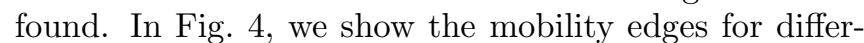
ent $\varepsilon_{A B}$. We note that the mobility edge for the usual Anderson model equals approximately \pm 6.2 at disorder $W=4$ [8, 33]. This agrees with the result for $\varepsilon_{A B}=0$ in Fig. 4

\section{Energy-concentration phase diagram}

Obviously, the values for $\Lambda_{M}$ and the mobility edges should strongly depend on the concentration. This is indeed the case as we show in Fig. 5 . Already a $10 \%$ difference in atomic composition leads to a pronounced asymmetry of the $\Lambda_{M}$ curves (compare, e.g., with Fig. 1). The energy-concentration phase diagram for $\varepsilon_{A B}=8$, and $W_{A}=W_{B}=4$ is presented in Fig. 6] For $x_{A}<$ $0.32\left(x_{B}>0.68\right)$, all electronic states of subband $A(B)$ are localized. Figs. [5] and [6] correspond to the case of overlapping bands such that the center of subband $A$ coincides with the edge of subband $B$ and vice versa.

\section{Concentration- $\varepsilon_{A B}$ phase diagram}

The concentration- $\varepsilon_{A B}$ phase diagram is presented in Fig. 7 for $E=\varepsilon_{A}=0$ and $W_{A}=W_{B}=4$. We see that the critical concentration is larger than the classical percolation threshold $x_{A}=0.3116$ for large $\varepsilon_{A B}$. We interpret this as being due to the localization effect in this quantum situation: a larger cluster of connected sites is needed to support extended states on sites of the sublattice $A$ (or $B$ ). For very large $\varepsilon_{A B}=100$ and $10^{3}$ (not shown) the critical concentration decreases to 0.416 and 0.384 , respectively, but remains larger than in the classical case. For large subband spacing sites with onsite potential $\varepsilon_{B}$ can be viewed as potential barriers to electrons from subband $A$ and vice versa. Thus electrons with energy $\approx \varepsilon_{A}$ have a low probability to hop onto $B$ sites and we are effectively studying the case of percolation. But even though $\varepsilon_{A B}$ might be large, there will always remain a finite but small probability for tunneling thus distinguishing our model from classical percolation. And since we are studying the wave equation (11), localization effects not present in classical percolation are important and lead to insulating behavior even when the concentration of sites supports a classically percolating cluster. Therefore, we find that the threshold for transport (extended states) in the quantum case is higher than for the classical one. We note that similar effects have been observed previously in studies of quantum percolation 34].

On the other hand, for small $\varepsilon_{A B}$, the critical concentration becomes less than the percolation threshold since tunneling between $A$ and $B$ sites is more significant. The phase diagram in Fig. 17 does not exactly show the mobility edges at which all extended states disappear for a given $\varepsilon_{A B}$, because it is determined at $E=0$. But as shown in Figs. [1] and [6] the $A$ band is not symmet- 


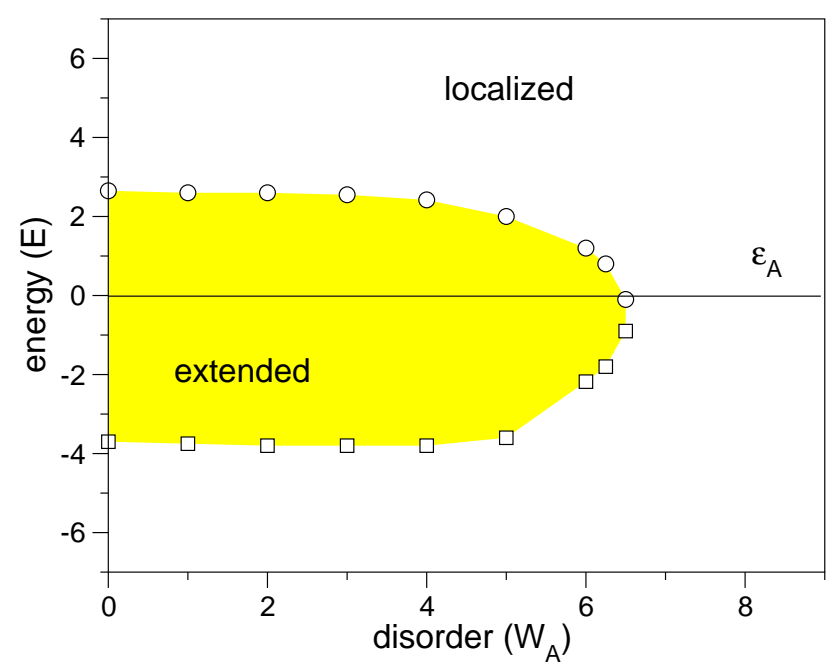

FIG. 3: The energy-disorder phase diagram for $\varepsilon_{A B}=17$ and $x_{A}=0.5$. The shaded region indicates extended states and the horizontal line denotes the value of $\varepsilon_{A}$. Here and in the following phase diagrams, $\square$ and $\circ$ indicate lower and upper mobility edges for the $A$ band, and, if included, upper and lower mobility edges for the $B$ band.

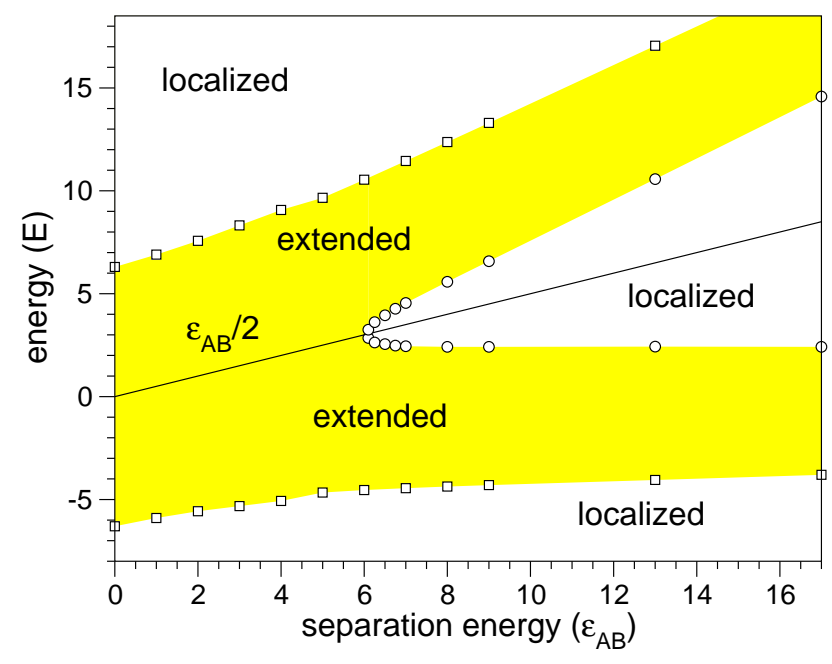

FIG. 4: The energy- $\varepsilon_{A B}$ phase diagram for $W_{A}=W_{B}=4$ and $x_{A}=0.5$. The shaded region indicates extended states. The upper $(B)$ and lower $(A)$ bands are symmetric with respect to $\varepsilon_{A B} / 2$ and the $B$ band has been constructed using this symmetry.

ric with respect to $\varepsilon_{A}(=0)$. As a consequence, more extended states occur for energies just below $\varepsilon_{A}$. This means that the phase boundary in Fig. 7 will be shifted slightly downwards, if $E$ is varied, too.

We note that an analogous phase diagram has been obtained recently [35] for the case of a two-dimensional binary alloy. The shape of the curve separating localized and extended electronic states is quite similar to our result shown in Fig. 7

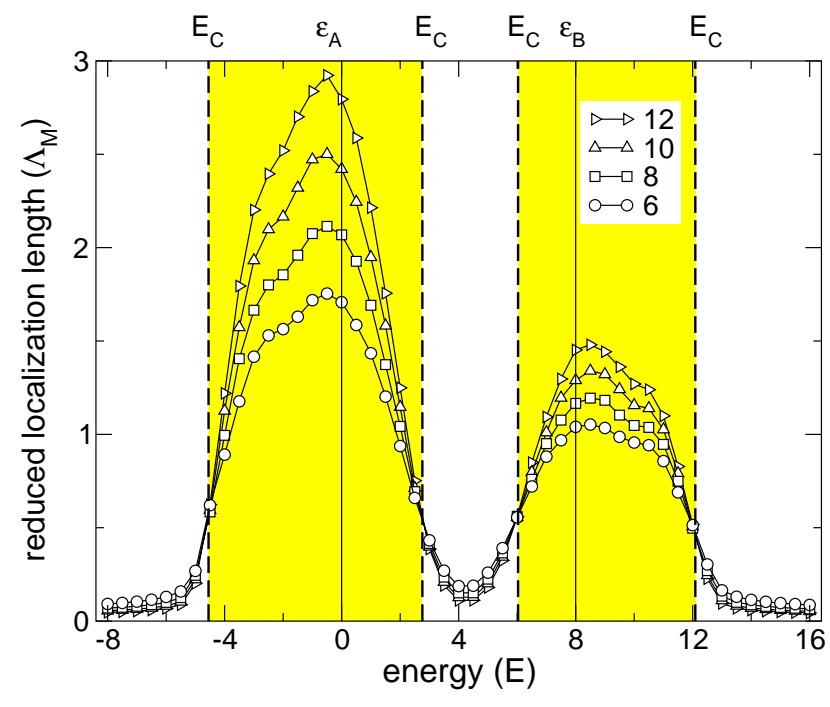

FIG. 5: The energy dependence of $\Lambda_{M}$ for a majority of $A$ sites (concentration $x_{A}=0.55$ ). System sizes are $M=6,8,10$ and $12, \varepsilon_{A B}=8$, and $W_{A}=W_{B}=4$. The two shaded regions indicate extended states. The solid and dashed vertical lines denote the band energies $\varepsilon_{A}$ and $\varepsilon_{B}$ and the position of the 4 mobility edges, $E_{\mathrm{c}}$, respectively.

\section{E. Disorder-concentration phase diagram}

The concentration dependence of the critical disorder - the disorder at which all states for a given energy become localized - is presented in Fig. 8 for energy $E=\varepsilon_{A}$. One can see that the critical disorder strongly depends on the concentration. For $x_{A}=1$, we recover the result $W_{\mathrm{c}}=16.5[8]$ of the single-band Anderson model. No extended electronic states are observed for 


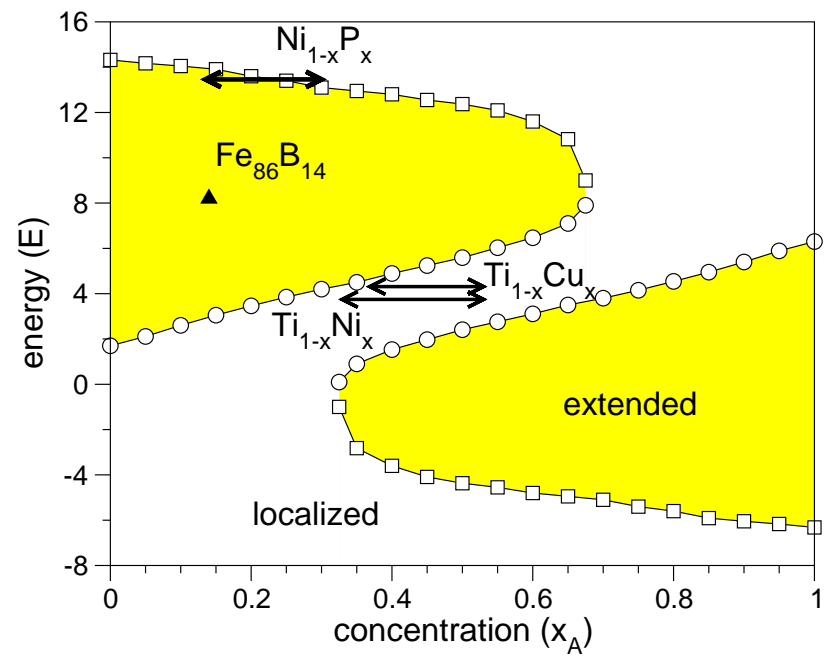

FIG. 6: The energy-concentration phase diagram at band separation $\varepsilon_{A B}=8$ and disorders $W_{A}=W_{B}=4$. The shaded regions denote extended states. The upper region of extended states has been constructed from point symmetry with respect to $\varepsilon_{A B} / 2$ and $x_{A}=0.5$. The thick solid lines and the triangle indicate the inferred concentration dependencies of 4 different AMA as discussed further in section IV

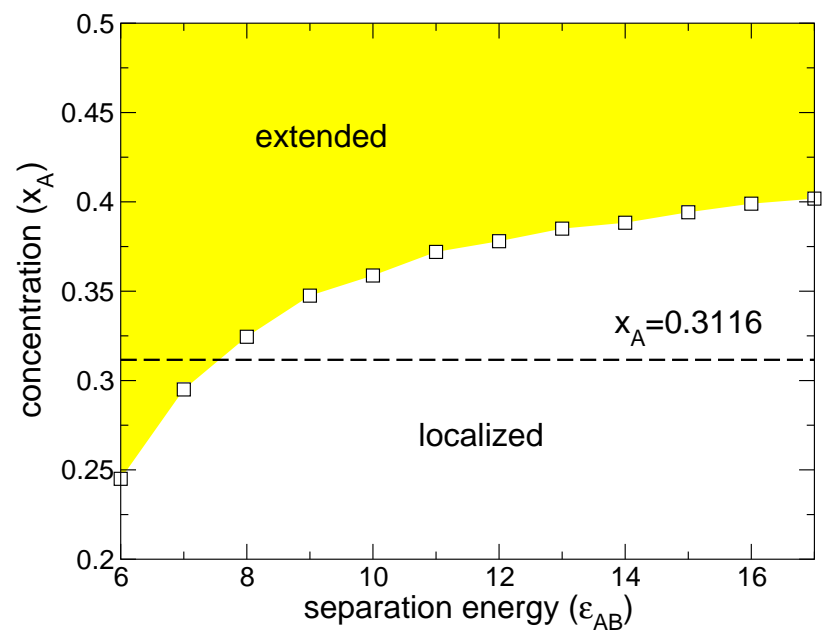

FIG. 7: The concentration- $\varepsilon_{A B}$ phase diagram at $E=\varepsilon_{A}=0$ and $W_{A}=W_{B}=4$. The shaded region denotes extended states. The dashed line represents the classical 3D sitepercolation threshold value $x_{A}=0.3116$.

$x_{A}<0.35$ in agreement with the idea that for small concentration the electronic states of these atoms form the usual localized donor subband of separated impurities.

For the concentration interval from 0.35 to 0.40 we obtain an additional transition as a function of disorder at small $W_{A}$ besides the usual Anderson MIT. For example, as shown in Fig. 9 for $x_{A}=0.38$ we observe localized behavior of $\Lambda_{M}(M)$ for small disorders. Increasing the disorder beyond $W_{A}>0.7$, we see extended states. At $W_{A} \sim 3.2$, the character of the states changes back to

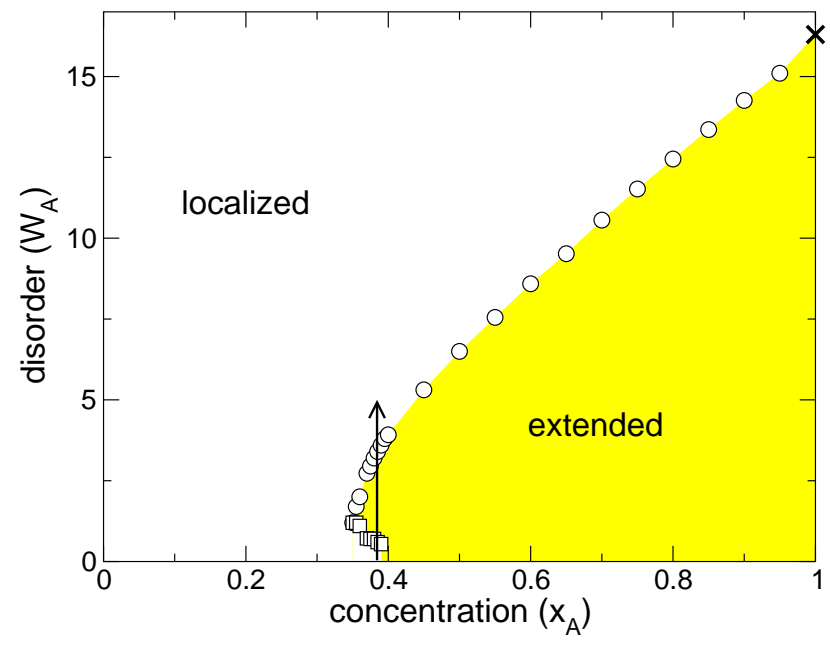

FIG. 8: The disorder-concentration phase diagram for $\varepsilon_{A B}=$ $17, W_{B}=4$, and $E=\varepsilon_{A}=0$. The shaded region denotes extended states. The arrow refers to the discussion in the text as well as to the data presented in Fig. 9] The $\times$ denotes the single-band result.

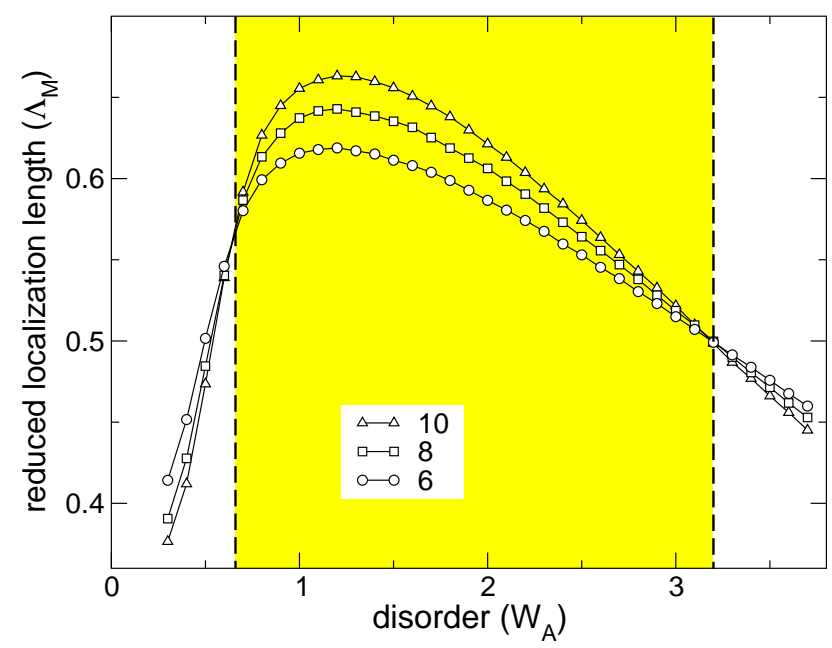

FIG. 9: The disorder dependence of $\Lambda_{M}$ for system sizes $M=$ 6,8 and 10 at $\varepsilon_{A B}=17, W_{B}=4, E=0$ and $x_{A}=0.38$. The shaded region indicates extended states and the two vertical lines denote approximate estimates of critical disorders $W_{\mathrm{c}}$.

localized. This behavior can be understood as follows: for $x_{A} \approx 0.38$ as indicated by the arrow in Fig. 8 the system at $W_{A}=0$ represents a binary alloy with large energy separation $\varepsilon_{A B}=17$. All states are localized, i.e., non-quantum percolating, on an already classically percolating set of sites $\{i(A)\}$ in the $A$ band. Small additional potential disorder will lead to an increase of certain $\varepsilon_{i(A)}$ as well as a decrease in certain $\varepsilon_{i(B)}$. A small change of the scattering conditions in these sites can then quickly allow enhanced transport across a now quantumpercolating backbone. But upon further increasing the disorder, the localization in each band will quickly lead 


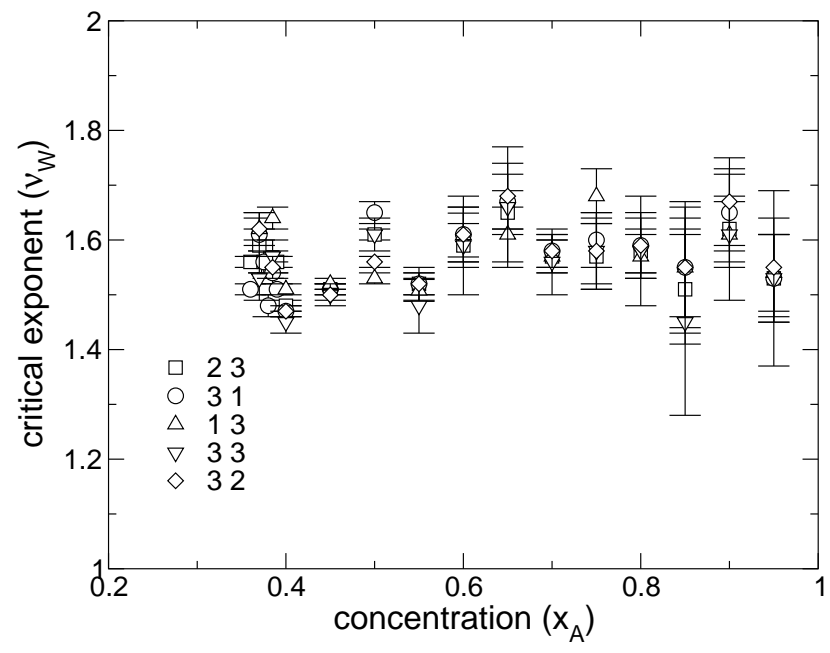

FIG. 10: Concentration dependence of the critical exponent $\nu_{W}$ obtained when varying the disorder across the mobility edge of Fig. 8 i.e., for $E=\varepsilon_{A}=0, \varepsilon_{A B}=17$ and $W_{B}=4$. The different symbols represent various orders $n_{r}$ and $m_{r}$ (indicated in the legend) of the expansion (4) used for the fitting. The error bars denote one standard deviation as obtained from the non-linear fitting procedure.

to localization again.

\section{F. Value of the critical exponent}

The critical exponent for the present model can be estimated by crossing the mobility edges as function of either disorder, energy or concentration, giving rise to the three exponents $\nu_{W}, \nu_{E}$ and $\nu_{x}$, respectively. Due to universality, their values should coincide with the critical exponent $\nu \approx 1.6$ of the single-band Anderson model since the universality class remains unchanged by the introduction of the binary disorder [36].

We have estimated the critical exponent $\nu$ for almost all points in the phase diagrams [3, 4, 6 , 7 and 8 as described in section [IC] For example, we compute $\nu_{W}$ along the mobility edge of the disorder-concentration phase diagram of Fig. 8. In Fig. [10 we show the resulting estimates when using different orders $n_{r}$ and $m_{r}$ for the fit function (44). As usual, the spread in values is somewhat larger than the least-square-error bars seem to suggest [32]. Summarizing the results, we find $\nu_{W}=1.56 \pm 0.06, \nu_{E}=1.64 \pm 0.05$ and $\nu_{x}=1.60 \pm 0.07$, compatible with $\nu \approx 1.6[37]$.

\section{DISCUSSION AND COMPARISON WITH EXPERIMENTS}

Let us now compare the above results of the binary Anderson model with experimental measurements of transport properties of AMA [22, 38]. Such a comparison will of course be purely qualitative. Nevertheless, it already suffices to understand much of the transport properties of such AMA. E.g., as mentioned in section [1 it is the position of the mobility edge relative to the value of the Fermi energy that determines the properties of transition-metal-based AMA. In this spirit, the Mooji correlation [2] is due to $E_{\mathrm{F}}$ being situated in a region of localized states, whereas the weak changes in resistivity at melting for $\mathrm{Fe}$ and $\mathrm{Co}$ as well as the positive temperature coefficient of resistivity for Fe-based AMA result from $E_{\mathrm{F}}$ being located in the center of the 3d-electronic band and hence, in the region of extended states.

\section{A. Transport properties of $\mathbf{N i}_{1-x} \mathbf{P}_{x}$}

The ionization energy of $\mathrm{P}$ is higher than that of $\mathrm{Ni}$, so our $A$ band corresponds to $\mathrm{P}$ atoms and the $B$ band to $\mathrm{Ni}$ atoms with $\varepsilon_{B}>\varepsilon_{A}$ as, e.g., shown in Fig. [6] According to the theoretical and experimental results of Ref. [39], $E_{\mathrm{F}}$ of the amorphous alloy $\mathrm{Ni}_{1-x} \mathrm{P}_{x}$ is located near the upper edge of the Ni-3d band (the $B$ band in our study). When the $\mathrm{P}$ concentration $x_{\mathrm{P}}$ increases from 0.15 to 0.27 the temperature coefficient of resistivity (TCR) decreases from positive to negative values [38]. The MIT is observed near $x_{\mathrm{P}}=0.25$. In Fig. [6] we indicated the corresponding region in the phase diagram. The twoband, binary random potential Anderson model can indeed qualitatively capture the observed experimental results. Even a quantitative comparison might be possible if a reliable way of obtaining values for $\varepsilon_{A}, \varepsilon_{B}$, and $W_{A}$ and $W_{B}$ becomes known. Note, however, that in the experiments, it is the hopping between $A$ and $B$ atoms at their respective random positions that leads to the broadening of the $A$ and $B$ bands and we have modeled this only qualitatively in the present work by the onsite disorders $W_{A}$ and $W_{B}$.

\section{B. Transport properties of $\mathbf{T i}_{1-x-y} \mathbf{C u}_{x} \mathbf{A}_{y}$ and $\mathbf{T} \mathbf{i}_{1-x-y} \mathbf{N} \mathbf{i}_{x} \mathbf{A}_{y}$}

A previous experimental study of the electronic structure of $\mathrm{Ti}_{1-x-y} \mathrm{Cu}_{x} \mathrm{~A}_{y}$ and $\mathrm{Ti}_{1-x-y} \mathrm{Ni}_{x} \mathrm{~A}_{y}$ amorphous alloys 22] (the symbol $\mathrm{A}_{y}$ represents additional admixtures) has shown that the valence band has two main peaks. The lower peak is formed predominantly by the $3 \mathrm{~d}$ states of $\mathrm{Cu}$ or $\mathrm{Ni}$ whereas the upper peak is due to the $3 \mathrm{~d}$ states of Ti. The Fermi level is located in the central region between the peaks and is hardly shifted upon changing the concentrations.

Within the present two-band model, this experimental situation may be modeled as shown in Fig. 6 The line for the $\mathrm{Ti}_{1-x-y} \mathrm{Ni}_{x} \mathrm{~A}_{y}$ alloys is situated somewhat below the one for $\mathrm{Ti}_{1-x-y} \mathrm{Cu}_{x} \mathrm{~A}_{y}$ because $\mathrm{Ni}$ has one electron less than $\mathrm{Cu}$. We believe that it is this difference that leads to the smaller value of the negative TCR for $\mathrm{Ti}_{1-x-y} \mathrm{Ni}_{x} \mathrm{~A}_{y}$ when compared to $\mathrm{Ti}_{1-x-y} \mathrm{Cu}_{x} \mathrm{~A}_{y}$. In both cases, $E_{\mathrm{F}}$ 
TABLE I: Temperature coefficient of resistivity $\alpha_{300}$ at $300 \mathrm{~K}$ for $\mathrm{Ti}_{1-x-y} \mathrm{Cu}_{x} \mathrm{~A}_{y}$ and $\mathrm{Ti}_{1-x-y} \mathrm{Ni}_{x} \mathrm{~A}_{y}$ amorphous alloys [22].

\begin{tabular}{l|c|l|c}
\hline \multicolumn{1}{c|}{ AMA } & $\begin{array}{c}\alpha_{300} \\
10^{-4} / \mathrm{K}\end{array}$ & \multicolumn{1}{c}{$\mathrm{AMA}$} & $\begin{array}{c}\alpha_{300} \\
\end{array}$ \\
\hline $\mathrm{Ti}_{62} \mathrm{Cu}_{33} \mathrm{P}_{5}$ & -0.70 & $\mathrm{Ti}_{70} \mathrm{Ni}_{25} \mathrm{Si}_{5}$ & -2.21 \\
$\mathrm{Ti}_{47} \mathrm{Cu}_{45} \mathrm{Ni}_{5} \mathrm{Si}_{3}$ & -1.11 & $\mathrm{Ti}_{60} \mathrm{Ni}_{36} \mathrm{P}_{2} \mathrm{Si}_{2}$ & -2.72 \\
$\mathrm{Ti}_{48} \mathrm{Cu}_{45} \mathrm{Ni}_{5} \mathrm{P}_{2}$ & -1.05 & $\mathrm{Ti}_{50} \mathrm{Ni}_{45} \mathrm{P}_{5}$ & -2.37 \\
$\mathrm{Ti}_{46} \mathrm{Cu}_{45} \mathrm{Ni}_{5} \mathrm{Si}_{2} \mathrm{P}_{2}$ & -3.03 & $\mathrm{Ti}_{45} \mathrm{Ni}_{50} \mathrm{P}_{5}$ & -3.28 \\
\hline
\end{tabular}

lies in a region of localized states in agreement with the negative TCR. In Table \ we show the values obtained for TCR.

Let us now analyze the concentration dependence of the TCR. When the Ti concentration decreases, the TCR also decreases as shown in Table \. This behavior can be explained from the concentration-energy phase diagram of Fig. [6 by the increase of the mobility edge $E_{\mathrm{c}}$. This in turn leads to an increasing distance between $E_{\mathrm{F}}$ and the region of unoccupied extended electronic states. The states close to $E_{\mathrm{F}}$ become more localized, leading to a more negative TCR.

Therefore, at least at a qualitative level, the present two-band Anderson model explains not only the negative TCR of $\mathrm{Ti}_{1-x-y} \mathrm{Cu}_{x} \mathrm{~A}_{y}$ and $\mathrm{Ti}_{1-x-y} \mathrm{Ni}_{x} \mathrm{~A}_{y}$ glasses but also the changes in TCR due to changes in composition $(\mathrm{Cu}$ or $\mathrm{Ni}$ ) and concentration. Furthermore, metallic glasses with a "metal"-like conductivity can be also treated within this model. For example, the Fermi level of $\mathrm{Fe}_{86} \mathrm{~B}_{14}$ alloy is situated in the region of extended states as indicated in Fig. 6 in agreement with its positive TCR 22.

\section{Overlapping the two bands}

Thus far, motivated by the experimental results, we concentrated on the case of separated bands, i.e., large $\varepsilon_{A B}$. To make the two regions of extended states in Fig. [6] overlap, we can in principle (i) keep $\varepsilon_{A B}$ fixed and change the disorder, or (ii) keep fixed disorder and decrease $\varepsilon_{A B}$ as in Fig. [4 From Fig. 3 we know that the position of mobility edges does not change a lot when decreasing $W_{A}$. On the other hand, increasing $W_{A}$ will lead to a narrower region of extended states in Fig. [6 and no overlap. Therefore, we choose to reduce $\varepsilon_{A B}$ in order to study what happens when the two bands overlap in the energy-concentration diagram.

In Fig. 11] we show such an overlap for $\varepsilon_{A B}=6$ and $W_{A}=W_{B}=4$. This situation corresponds to large overlap of the two subbands. We see that the shape of the regions of extended states remains nearly unchanged. This leads to non-monotonicity in the behavior of the upper and lower mobility edges. We remark that in order to resolve this behavior, the accuracy of the TMM had to

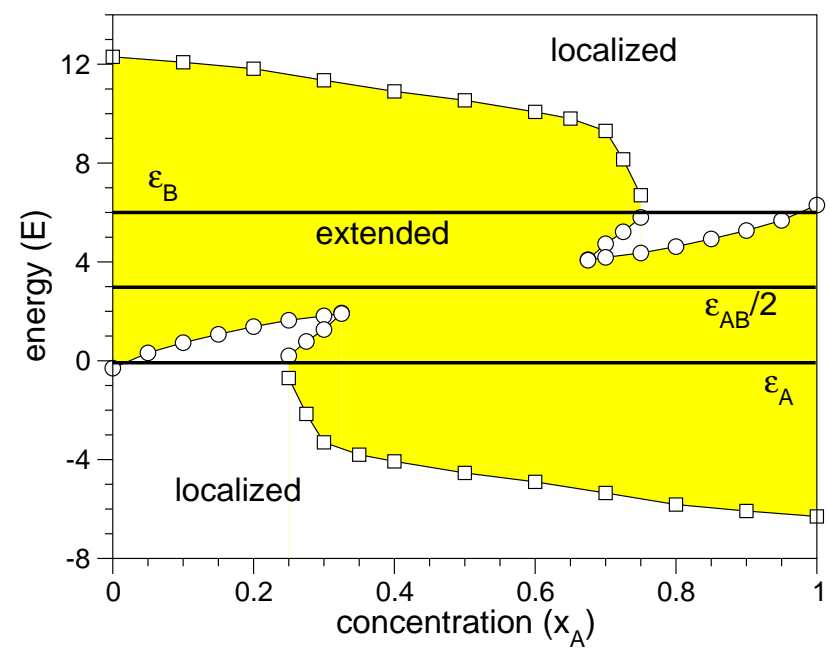

FIG. 11: The energy-concentration phase diagram at band separation $\varepsilon_{A B}=6$ and disorders $W_{A}=W_{B}=4$. The shaded region denotes extended states. The horizontal lines indicate $\varepsilon_{B}, \varepsilon_{A B} / 2$ and $\varepsilon_{B}$ from top to bottom, respectively. The upper mobility edge has been constructed from point symmetry with respect to $\varepsilon_{A B} / 2$ and $x_{A}=0.5$.

be increased significantly up to very small errors of $0.05 \%$ for the localization lengths. We emphasize that a similar accuracy was needed to resolve the non-monotonicity in the disorder-concentration diagram in Fig. 8

When further decreasing $\varepsilon_{A B}$ to 5 , the behavior of the mobility edges becomes regular again and their values eventually reduce to the values of the well-known phase diagram of the single-band Anderson model [40].

\section{CONCLUSIONS}

We have shown that the three-dimensional Anderson model of localization with binary random potential disorder allows to explain not only various peculiarities of the transition-metal-based amorphous alloys but also how these change as the chemical composition changes. Even for a comparatively large overlap of the two subbands, a region of localized electronic states exists in the central part between the two subbands. This confirms the assumption made in Ref. 22 for the experimental results in AMA. Even a quantitative analysis, using suitably extracted parameters from density-of-states measurements appears possible. Of course in this case ternary and quaternary AMA will have to be distinguished by additional onsite potentials similar to $\varepsilon_{A}$ and $\varepsilon_{B}$ for the binary model.

In addition to the usefulness of the two-band Anderson model for a qualitative comparison with experiments, there is also the interesting fact that the model allows to study the concentration dependence and the associated MIT. This concentration dependence is clearly a prominent feature present in most disordered materials. We 
find that, as expected from universality, the universality class of this additional transition remains unchanged with respect to a single-band Anderson model.

\section{Acknowledgments}

We thank A. Eilmes, S. Klassert and P. Stollmann for useful comments. This work was supported by grants from the DAAD via Ref. 322, A/01/20012 and the DFG via SFB393 .
[1] R. Hasegawa, Glassy Metals: magnetic, chemical and structural properties (CRC Press, New York, 1983).

[2] J. Mooij, Phys. Status Solidi A 17, 521 (1973).

[3] P. A. Lee and T. V. Ramakrishnan, Rev. Mod. Phys. 57, 287 (1985).

[4] P. Cote and L. Meisel, Phys. Rev. B 25, 2138 (1982).

[5] L. Meisel and P. Cote, Phys. Rev. B 30, 1743 (1984).

[6] P. W. Anderson, Phys. Rev. 109, 1492 (1958).

[7] D. Belitz and T. R. Kirkpatrick, Rev. Mod. Phys. 66, 261 (1994).

[8] B. Kramer and A. MacKinnon, Rep. Prog. Phys. 56, 1469 (1993).

[9] N. W. Ashcroft and N. D. Mermin, Solid State Physics (Saunders College, New York, 1976).

[10] E. Abrahams, P. W. Anderson, D. C. Licciardello, and T. V. Ramakrishnan, Phys. Rev. Lett. 42, 673 (1979).

[11] Y. Imry, Phys. Rev. Lett. 44, 469 (1979).

[12] M.-A. Park, K. Savran, and Y.-J. Kim, Supercond. Sci. Technol. 14, L31 (2001).

[13] O. Madelung, Introduction to solid state theory (Springer, Berlin Heidelberg New York, 1978).

[14] B. Hickey, D.Greig, and M. Howson, J.Phys.F:Met.Phys. 16, L13 (1986).

[15] H. Boghosian and M. Howson, Phys. Rev. B 41, 7397 (1990).

[16] M. Howson, B.J.Hickey, and G.J.Morgan, Phys. Rev. B 38, 5267 (1988).

[17] J. Bieri, A. Fert, G. Creuzet, and J. Ousset, Solid State Commun. 49, 849 (1984).

[18] A. Seeger, P.Lunnkenheimer, J. Hemberger, and et al., J. Phys.: Condens. Matter 11, 3273 (1999).

[19] M. Howson and D.Greig, J.Phys.F:Met.Phys. 16, 989 (1986).

[20] R. Rosenbaum, A. Heines, A. Palevski, M. Karpovski, A. Gladkikh, M. Pilosof, A. J. Daneshvar, M. R. Graham, T. Wright, J. T. Nicholls, C. J. Adkins, M. Witcomb, V. Prozesky, W. Przybylowicz, and R. Pretorius, J. Phys.:
Condens. Matter 9, 5395 (1997).

[21] C. Tsuei, Phys. Rev. Lett. 57, 1943 (1986).

[22] O. I. Nakonechnaya, I. V. Plyushchai, M. P. Semen'ko, and N. I. Zakharenko, Physics of Metals and Metallography 90, 439 (2000).

[23] H. Grussbach and M. Schreiber, Phys. Rev. B 57, 663 (1995).

[24] J. Hafner, J. Phys.: Condens. Matter 3, F23 (1991).

[25] J.-L. Pichard and G. Sarma, J. Phys. C 14, L127 (1981).

[26] A. MacKinnon and B. Kramer, Phys. Rev. Lett. 47, 1546 (1981).

[27] A. MacKinnon and B. Kramer, Z. Phys. B 53, 1 (1983).

[28] I. Zambetaki, Q. Li, E. N. Economou, and C. M. Soukoulis, Phys. Rev. Lett. 76, 3614 (1996), ArXiv: cond-mat/9704107

[29] D. J. Thouless, Phys. Rep. 13, 93 (1974).

[30] K. Slevin and T. Ohtsuki, Phys. Rev. Lett. 82, 382 (1999), ArXiv: cond-mat/9812065

[31] A. MacKinnon, J. Phys.: Condens. Matter 6, 2511 (1994).

[32] F. Milde, R. A. Römer, and M. Schreiber, Phys. Rev. B 61, 6028 (2000).

[33] B. Bulka, M. Schreiber, and B. Kramer, Z. Phys. B 66, 21 (1987).

[34] Y. Avishai and J. Luck, Phys. Rev. B 45, 1074 (1992).

[35] H. Nazareno, P. de Brito, and E. Rodrigues, Phys. Rev. B 66, 012205 (2002).

[36] E. Hofstetter and M. Schreiber, Europhys. Lett. 21, 933 (1993).

[37] F. Milde, R. A. Römer, M. Schreiber, and V. Uski, Eur. Phys. J. B 15, 685 (2000), ArXiv: cond-mat/9911029

[38] P. Cote, Solid State Commun. 18, 1311 (1976).

[39] N. Babich, N. Poperenko, I. Shaykevich, and N. Zakharenko, Optical Materials 4, 617 (1995).

[40] B. Bulka, B. Kramer, and A. MacKinnon, Z. Phys. B 60, 13 (1985). 